\title{
Descending necrotizing mediastinitis after sequestrectomy in a patient with bisphosphonate-induced osteonecrosis of the jaw: A case report
}

\author{
TAMAKI TAMANUKI ${ }^{1}$, TOMOYOSHI AOYAGI ${ }^{1}$, AKIYUKI MURANO $^{2}$ and HIROSHI MATSUZAKI ${ }^{1}$ \\ Departments of ${ }^{1}$ Breast Surgery, and ${ }^{2}$ Oral and Maxillofacial Surgery, \\ Funabashi Municipal Medical Center, Funabashi, Chiba 273-8588, Japan
}

Received December 28, 2017; Accepted June 11, 2018

DOI: $10.3892 / \mathrm{mco} .2018 .1656$

\begin{abstract}
An 83-year-old woman underwent mastectomy for breast cancer of the right breast in 2008. In addition to hormone therapy and irradiation, zoledronate was started for bone metastasis 6 months postoperatively. Five years after the operation, the patient developed osteonecrosis of the jaw, and underwent sequestrectomy because of uncontrollable pain in the mandible. The patient visited our hospital for a 1-week history of fever and right facial swelling with pain, and was diagnosed with right mandibular cellulitis. Despite antibiotic therapy, the patient fell into shock. Follow-up computed tomography showed gas formation extending down to the posterior mediastinum, which was compatible with descending necrotizing mediastinitis (DNM). The patient succumbed to septicemia on the third hospital day. The mortality rate of DNM greatly increases in patients with advanced cancer because clinicians cannot perform radical treatment due to the impaired general condition and limited life expectancy. DNM advances by the hour; therefore, repeated computed tomography is essential when antibiotic therapy does not improve the patient's condition. Attention must be paid to detect signs of DNM in such patients. To the best of our knowledge, this is the first report in English regarding DNM caused by bisphosphonate-induced osteonecrosis of the jaw.
\end{abstract}

\section{Introduction}

Bisphosphonate (BP) is used for bone metastasis or hormonal agent-related bone loss in patients with breast cancer (1-4). The mechanism of BP involves inhibiting the synthesis and liberation of osteoclasts, which results in promoting resistance

Correspondence to: Dr Tamaki Tamanuki, Department of Breast Surgery, Funabashi Municipal Medical Center, 1-21-1 Kanasugi, Funabashi, Chiba 273-8588, Japan

E-mail: tamaki38tamaki@yahoo.co.jp

Key words: breast cancer, bone metastasis, bisphosphonate, osteonecrosis of the jaw, descending necrotizing mediastinitis against bone resorption $(5,6)$. Therefore, long-term use of BP easily causes osteoporotic fractures (7). In 2003, osteonecrosis of the jaw (ONJ) was reported as a serious complication of BP treatment for the first time (8).

Odontogenic infection can spread from the submandibular space to the retropharyngeal space, and lead to descending necrotizing mediastinitis (DNM). DNM progresses rapidly, without specific clinical findings, and requires invasive procedures, including mediastinal drainage or open thoracic surgery. Thus, it is associated with high mortality, even in this antibiotic era. Once BP-induced ONJ causes mandibular osteomyelitis, it may potentially lead to DNM. To the best of our knowledge, however, DNM caused by BP-induced ONJ have never been reported before. This is the first case in the English literature.

Here, we present a rare case of DNM caused by BP-induced ONJ in a patient with bone metastasis of breast cancer. It is difficult to manage DNM in patients with terminal cancer because of their impaired general condition and limited longevity. Therefore, we should start prophylactic treatment and detect the initial signs of DNM carefully.

\section{Case report}

An 83-year-old woman was referred to our hospital for a mass with skin distortion in her right breast 9 years ago. At this time, the patient was diagnosed with estrogen receptor-positive breast cancer and axillary lymph node metastasis, and underwent right mastectomy and axillary lymphadenectomy. Of the 14 lymph nodes identified in the axillary tail, 8 were positive for malignancy. Pathological examination revealed pT3N1M0. Bone metastasis was found in the right costal bone 6 months after the operation, despite treatment with letrozole concomitant with irradiation postoperatively. Therefore, we added zoledronate. The patient had no documented history of dental treatment before BP treatment. In addition, serum tumor marker levels gradually elevated. We changed the regimen to high-dose toremifene (12 mg/day) due to lung metastasis. However, the patient's condition worsened despite modifying the regimen of these hormonal agents.

Bone scintigraphy showed abnormal uptake in the mandibular bone 5 years after the operation. We diagnosed ONJ and 
treated the patient conservatively with oral administration of cefcapene pivoxil for 3 years and 8 months. Four months prior to the present referral, however, the patient underwent sequestrectomy because the ONJ involved the mandibular canal and the patient presented with uncontrollable pain. First, oral mucosa around the exposed sequestrum was incised, and mucoperiosteal flap tissue was dissected. Following exposure of the borderline between the normal bone and sequestrum, the anterior portion was removed completely. Next, the remaining posterior lesion was removed as much as possible to avoid injury to arteries in the mandible because the laboratory data showed anemia. At this time, cefazolin was administered perioperatively. Additional sequestrectomy for the residual lesion was planned after anemia improved. Four years and 6 months after the initiation of BP therapy, the patient developed osteonecrosis of the jaw. Therefore, we stopped BP immediately and did not resume administration. In addition, the patient underwent no other dental procedures except for sequestrectomy while she had treatment for cancer. BP was administered for 4 years and 6 months in total, including 2 cessation periods at 2 and 5 months for transient impairment of renal function.

The patient presented to the emergency department with a 1-week history of fever, dyspnea, and right facial swelling with pain. Blood tests showed an elevated white blood cell count of $25,500 / \mu 1$ and C-reactive protein level of $14.05 \mathrm{mg} / \mathrm{dl}$. Computed tomography (CT) demonstrated inflammation in the right mandibular subcutaneous tissue, but no gas formation or airway obstruction (Fig. 1). The patient was diagnosed with right mandibular cellulitis. The patient was admitted to our hospital and we started intravenous infusion of ceftriaxone. Inflammatory marker levels continued to rise despite antibiotic therapy. The patient's general condition worsened rapidly, and she fell into shock. We performed repeated neck and chest CT scans, and found de novo gas formation extending to the cervical and retropharyngeal spaces, down to the posterior mediastinum (Fig. 2A-C). Consequently, the patient was diagnosed with DNM, and surgical drainage was considered. However, we could not perform any further invasive procedures because of the patient's impaired general condition and her family's wishes. The patient died of septicemia on the third hospital day. Fig. 3 shows the entire clinical course for the treatment of breast cancer.

\section{Discussion}

BP-induced ONJ is a rare, but serious complication, that reduces quality of life considerably. Bamias et al (9) reported that the incidence of BP-induced ONJ in patients with breast cancer was $2.9 \%(2 / 70)$, whereas that in all patients with BP treatment was $6.7 \%$ (17/252). Periodontal disease and a history of tooth extraction, as well as various cancer treatments, including hormone, radiation, and chemotherapies, can increase the risk for ONJ $(6,10)$. In this case, a 4-year history of BP treatment and a 9-year history of hormone therapy predisposed the patient to ONJ.

In general, BP-induced ONJ is treated with antibiotic therapy and cessation of BP. Increased apoptosis of keratinocytes impairs the oral mucosal barrier in patients with BP treatment $(6,10)$. Therefore, it is preferable to avoid any surgical procedures as an initial treatment for either diagnostic

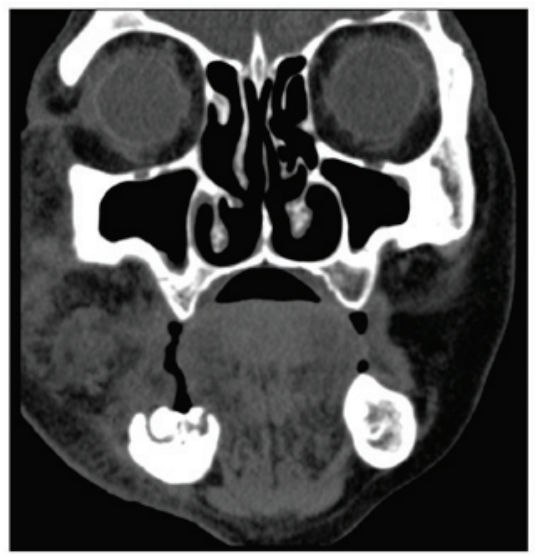

Figure 1. Computed tomography on admission. Inflammation in the right mandibular subcutaneous tissue and soft tissue edema was indicated, but neither gas formation nor airway obstruction was demonstrated.

or therapeutic purposes $(5,6,8)$. The present patient was closely followed up after the diagnosis of ONJ. However, we performed sequestrectomy to manage uncontrollable pain. This surgical intervention might potentially be the cause of the osteomyelitis and subsequent mediastinitis. There is no clear guideline that indicates sequestrectomy for ONJ. As this case implied, however, we should avoid sequestrectomy as long as possible, and perform careful follow-up of elderly patients and those with poor general health status if they receive long-term BP treatment.

In our institute, regardless of age, BP is administered in patients with bone metastasis as long as renal function does not worsen. In patients with a history of dental procedures, we consult with the oral and maxillofacial surgeon as to whether BP can be administered. We also consult the oral surgeon to decide cessation of BP therapy when symptoms of osteonecrosis of the jaw are observed. The more BP accumulates in the body, the higher the risk of ONJ. The risk drastically increases when the administration period exceeds 4 years in total (11). Some clinicians have reported that as many as 50\% of patients with a malignant tumor have inflammatory dental disease including periodontal disease before the development of BP-induced ONJ (12). It is also thought that the direct predisposing factor is not the invasiveness of the procedure itself, but the local inflammation that led to the surgical procedure. Therefore, it is important to confirm that the oral environment is healthy before starting BP therapy. Moreover, patients with long-term BP therapy require regular follow-up of serum calcium level and use of prophylactic antibiotics for dental procedures.

The most common causes of mediastinitis are esophageal perforation and surgery with sternotomy (13). DNM, which is a rare type of mediastinitis, occurs as a complication of odontogenic or cervicofascial infections spreading down the deep fascial planes into the mediastinum (14). The mortality rate reaches $50 \%$, even in the current antibiotic era (15). A recent review estimated the mortality rate at $25 \%$ (16), and DNM can thus be regarded as one of the most complicated infections. Predisposing factors previously reported are age over 70 years, alcoholism, and diabetes mellitus (14). In addition, the incidence of DNM is much higher in patients who are in poor 

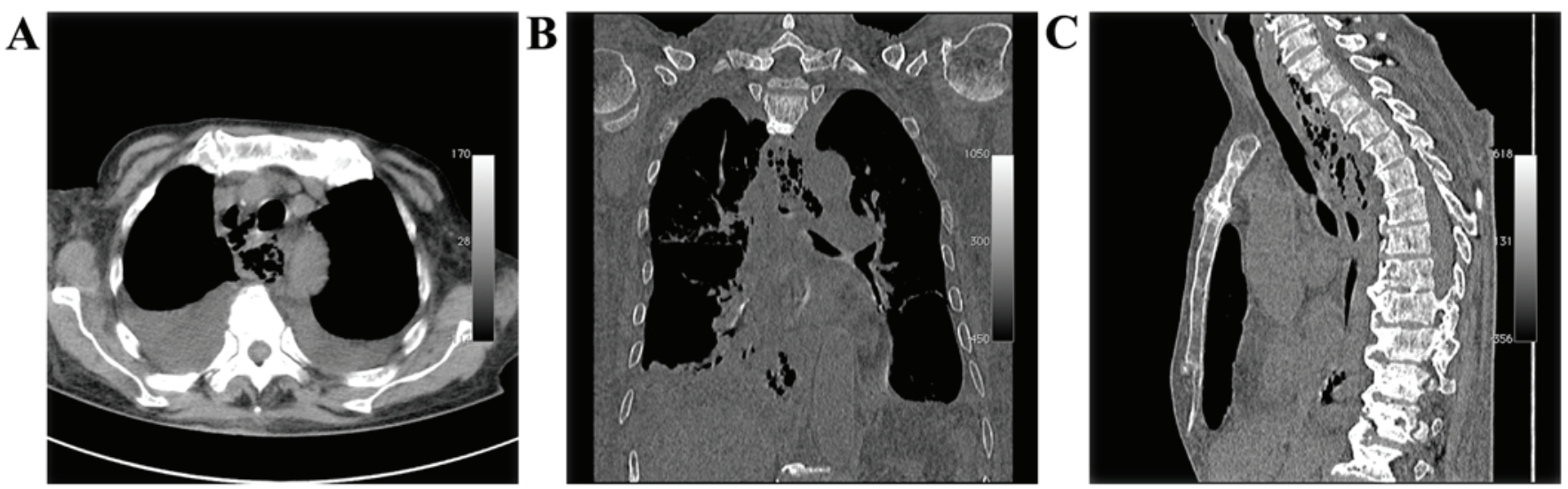

Figure 2. Cervical and chest computed tomography 2 days after admission. De novo gas formation extending to the cervical and retropharyngeal spaces down to the posterior mediastinum was indicated. (A) axial, (B) coronal and (C) sagittal view.

\section{LET+Ca TOR}

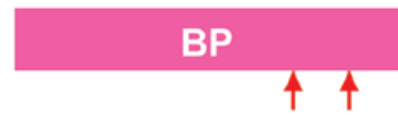

\section{cefcapene pivoxil}

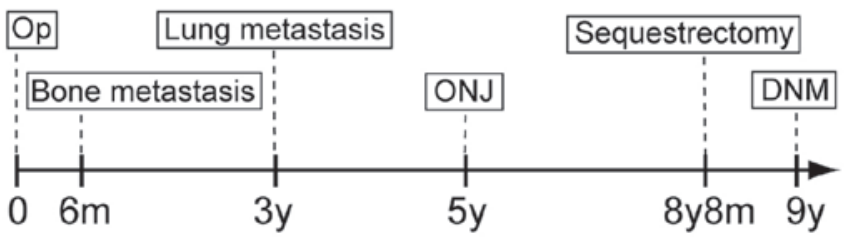

Figure 3. Entire clinical course for the treatment of breast cancer. Red arrows show cessation periods ( 2 and 5 months, respectively). BP, Bisphosphonate; DNM, Descending necrotizing mediastinitis; LET, Letrozole; m, Month; ONJ, Osteonecrosis of the jaw; Op, Operation (mastectomy); TOR, Toremifene; y, Year.

physical condition owing to malnutrition, drug addiction, or immunosuppression (17).

Maxillofacial infection can spread into the mediastinum through the following routes $(13,14)$ : i) Pretracheal space: The infection descends into the frontal mediastinum through the suprapleural membrane (Sibson's fascia). ii) Retropharyngeal space: This route begins from the base of the skull passing downwards into the posterior mediastinum, thereby enabling direct access of the dental infection to the mediastinum. iii) Perivascular space: This route is a vascular sheath for the great cervical vessels-the carotid artery and internal jugular vein.

According to Moncada et al (18), $71 \%$ of cervical infections spread through retropharyngeal space, while $21 \%$ of cases descend through perivascular space. In the present case, the focus of infection was a necrotic lesion in the mandibular canal, which communicates with the dental alveolus. Thus, we speculated that the infection extended down the retropharyngeal space.

The gold standard for diagnosis of DNM is CT, which can clearly detect inflammation, abscesses, and gas forma- tion $(14,16)$. In the present case, CT on admission revealed evidence of inflammation in subcutaneous tissue of the right mandibular bone, but no gas formation. Therefore, the patient was initially diagnosed with cellulitis. However, considerable gas formation was seen from the mandibular bone to the posterior mediastinum on CT 2 days later. This finding was compatible with DNM. The mortality associated with DNM cannot be reduced drastically, even with antibiotic therapy and drainage, while a delay of diagnosis and incomplete drainage cause further increased mortality $(13,17)$. Most cases of DNM are caused by odontogenic infection in the roots of the second and third lower molars, because these teeth are directly communicated with the submandibular space. Therefore, infection from these teeth descends into the mediastinum more rapidly and diffusely. A conservative surgical approach is insufficient to manage DNM, and it requires more radical drainage, including open chest surgery $(14,17)$. We considered performing mediastinal drainage, in addition to antibiotic therapy. However, it was impossible to perform surgical intervention because the patient's general condition was severely impaired by her progressive cancer.

In conclusion, once BP-induced ONJ in patients with advanced cancer causes DNM, the mortality risk greatly increases because clinicians cannot perform radical treatment due to the impaired general condition and limited life expectancy. We should perform CT and check the current condition while keeping the possibility of DNM in mind when patients with BP-induced ONJ present trismus, dysphagia, and maxillofacial edema. To prevent progression, antibiotic therapy should be started empirically, regardless of the presence of findings implying DNM. Subsequently, aggressive surgical drainage should be performed as soon as abscess formation is found. As this case showed, DNM advances by the hour. Repeated CT scans are essential when antibiotic therapy does not improve the patient's condition. Attention must be paid to detect signs of DNM in such patients.

\section{Acknowledgements}

The authors would like to thank Dr Tomoki Sakata (Department of Cardiovascular Surgery, Funabashi Municipal Medical Center) for valuable advice on mediastinitis. 


\section{Funding}

No funding was received.

\section{Availability of data and materials}

The datasets used and/or analyzed during the current study are available from the corresponding author on reasonable request.

\section{Authors' contributions}

TT made substantial contributions to conception and design of the study and wrote the manuscript. TA, AM, and HM participated in the treatment of this patient and provided supervision of this manuscript editing and revised each draft regarding ONJ (AM) and breast cancer (TA and HM). All authors read and approved the final manuscript.

\section{Ethics approval and consent to participate}

Not applicable.

\section{Patient consent for publication}

Patient consent for publication of data was obtained.

\section{Competing interests}

The authors declare that they have no competing interests.

\section{References}

1. Kohno N, Aogi K, Minami H, Nakamura S, Asaga T, Iino Y, Watanabe T, Goessl C, Ohashi Y and Takashima S: Zoledronic acid significantly reduces skeletal complications compared with placebo in Japanese women with bone metastases from breast cancer: A randomized, placebo-controlled trial. J Clin Oncol 23: 3314-3321, 2005

2. Takahashi S,Iwase T,Kohno N,IshikawaT, Taguchi T, Takahashi M, Horiguchi J, Nakamura S, Hozumi Y, Fukunaga M and Noguchi S Efficacy of zoledronic acid in postmenopausal Japanese women with early breast cancer receiving adjuvant letrozole: 12-month results. Breast Cancer Res Treat 133: 685-693, 2012.

3. Mauri D, Valachis A, Polyzos IP, Polyzos NP, Kamposioras K and Pesce LL: Osteonecrosis of the jaw and use of bisphosphonates in adjuvant breast cancer treatment: A meta-analysis. Breast Cancer Res Treat 116: 433-439, 2009.

4. Valachis A, Polyzos NP, Georgoulias V, Mavroudis D and Mauri D: Lack of evidence for fracture prevention in early breast cancer bisphosphonate trials: A meta-analysis. Gynecol Oncol 117: 139-145, 2010.

5. Marx RE, Sawatari Y, Fortin $M$ and Broumand V: Bisphosphonate-induced exposed bone (osteonecrosis/osteopetrosis) of the jaws: Risk factors, recognition, prevention, and treatment. J Oral Maxillofac Surg 63: 1567-1575, 2005.
6. Kyrgidis A, Vahtsevanos K, Koloutsos G, Andreadis C Boukovinas I, Teleioudis Z, Patrikidou A and Triaridis S: Bisphosphonate-related osteonecrosis of the jaws: A case-control study of risk factors in breast cancer patients. J Clin Oncol 26: 4634-4638, 2008.

7. Kim YS and Park WC: Atypical subtrochanteric femur fracture in patient with metastatic breast cancer treated with zoledronic acid. J Breast Cancer 15: 261-264, 2012.

8. Marx RE: Pamidronate (Aredia) and zoledronate (Zometa) induced avascular necrosis of the jaws: A growing epidemic. J Oral Maxillofac Surg 61: 1115-1117, 2003.

9. Bamias A, Kastritis E, Bamia C, Moulopoulos LA, Melakopoulos I, Bozas G, Koutsoukou V, Gika D, Anagnostopoulos A, Papadimitriou C, et al: Osteonecrosis of the jaw in cancer after treatment with bisphosphonates: Incidence and risk factors. J Clin Oncol 23: 8580-8587, 2005.

10. Vahtsevanos K, Kyrgidis A, Verrou E, Katodritou E, Triaridis S, Andreadis CG, Boukovinas I, Koloutsos GE, Teleioudis Z, Kitikidou $\mathrm{K}$, et al: Longitudinal cohort study of risk factors in cancer patients of bisphosphonate-related osteonecrosis of the jaw. J Clin Oncol 27: 5356-5362, 2009.

11. Ruggiero SL, Dodson TB, Assael LA, Landesberg R, Marx RE and Mehrotra B; American Association of Oral and Maxillofacial Surgeons: American Association of Oral and Maxillofacial Surgeons position paper on bisphosphonate-related osteonecrosis of the jaws-2009 update. J Oral Maxillofac Surg 67 (Suppl 1): S2-S12, 2009.

12. Qi WX, Tang LN, He AN, Yao Y and Shen Z: Risk of osteonecrosis of the jaw in cancer patients receiving denosumab: A meta-analysis of seven randomized controlled trials. Int J Clin Oncol 19: 403-410, 2014.

13. Papalia E, Rena O, Oliaro A, Cavallo A, Giobbe R, Casadio C, Maggi $G$ and Mancuso M: Descending necrotizing mediastinitis: Surgical management. Eur J Cardiothorac Surg 20: 739-742, 2001.

14. Novakov IP, Safev GP and Peicheva SE: Descending necrotizing mediastinitis of odontogenic origin-personal experience and literature review. Folia Med (Plovdiv) 52: 13-20, 2010.

15. Sancho LM, Minamoto H, Fernandez A, Sennes LU and Jatene FB: Descending necrotizing mediastinitis: A retrospective surgical experience. Eur J Cardiothorac Surg 16: 200-205, 1999.

16. Furst IM, Ersil P and Caminiti M: A rare complication of tooth abscess-Ludwig's angina and mediastinitis. J Can Dent Assoc 67: 324-327, 2001.

17. Chen KC, Chen JS, Kuo SW, Huang PM, Hsu HH, Lee JM and Lee YC: Descending necrotizing mediastinitis: A 10-year surgical experience in a single institution. J Thorac Cardiovasc Surg 136: 191-198, 2008.

18. Moncada R, Warpeha R, Pickleman J, Spak M, Cardoso M, Berkow A and White H: Mediastinitis from odontogenic and deep cervical infection. Anatomic pathways of propagation. Chest 73: 497-500, 1978. 